\title{
Analyzing Homogeneity and Heterogeneity of Change Using Rasch and Latent Class Models: A Comparative and Integrative Approach
}

\author{
Thorsten Meiser \\ University of Heidelberg \\ Monika Hein-Eggers, Pamela Rompe, and Georg Rudinger \\ University of Bonn
}

The application of unidimensional Rasch models to longitudinal data assumes homogeneity of change over persons. Using latent class models, several classes with qualitatively distinct patterns of development can be taken into account; thus, heterogeneity of change is assumed. The mixed Rasch model integrates both the Rasch and the latent class approach by dividing the population of persons into classes that conform to Rasch models with class-specific parameters. Thus, qualitatively different patterns of change can be modeled with the homogeneity assumption retained within each class, but not between classes. In contrast to the usual latent class approach, the mixed Rasch model includes a quantitative differentiation among persons in the same class. Thus, quantitative differences in the level of the latent attribute are disentangled from the qualitative shape of development. A theoretical comparison of the formal approaches is presented here, as well as an application to empirical longitudinal data. In the context of personality development in childhood and early adolescence, the existence of different developmental trajectories is demonstrated for two aspects of personality. Relations between the latent trajectories and discrete exogenous variables are investigated. Index terms: latent class analysis, latent structure analysis, measurement of change, mixture distribution models, Rasch model, rating scale model.

Latent structure analysis (LSA) is a methodological framework for modeling and testing hypotheses about the occurrence and the source of multivariate associations in multidimensional contingency tables. LSA models characterize the probabilities of manifest response vectors by an assumed unidimensional latent person characteristic $\theta$ or an assumed $k$-dimensional latent person vector $\theta=\left(\theta_{1}, \ldots, \theta_{k}\right)$ and a set of structural item parameters (Andersen, 1982, 1988, 1990; Clogg, 1988; Lazarsfeld, 1968; Lazarsfeld \& Henry, 1968).

Because of conditional stochastic independence of responses given a value of the latent variable or vector, the multivariate associations among the manifest indicators are reduced to their common dependence on the latent variable for a unidimensional latent space, and to their dependence on the latent variables and the associations between the latent variables if $\theta$ is multidimensional. In the general view proposed here, the LSA approach is the joint super model for unidimensional latent trait and latent class models, for multidimensional latent trait and latent class models, as well as for multidimensional latent structure models with both continuous and discrete latent variables. Common as well as distinctive features of latent trait and latent class models are described by Langeheine \& Rost (1988) and in the extended review by Andrich (1991). The present paper focuses on a family of LSA models that is based on the assumption of a two-dimensional latent vector, with 
one discrete and one continuous latent variable.

In probabilistic test theory, the goal of most LSA applications is to explain the associations within a set of discrete test items by a common latent variable (i.e., to achieve unidimensionality of the items in terms of test construction or evaluation). Thus, the elimination of differential item functioning (DIF) is of crucial importance. DIF is defined as any variation of the item-specific category probabilities due to manifest or latent factors other than the (mostly unidimensional) latent attribute intended to be measured by the test.

Numerous generalizations of LSA models have made LSA a flexible method for the analysis of discrete indicators that is comparable to the family of linear structural equation models for the multivariate analysis of continuous indicators. Major contributions to this development have been the incorporation of several latent variables as latent vectors (e.g., Andersen, 1985, 1988, 1991; Collins \& Wugalter, 1992; Duncan, 1983, 1984; Embretson, 1991; Goodman, 1974a, 1974b; Hagenaars, 1988; Kelderman, 1992, 1993; Kelderman \& Rijkes, 1994; Langeheine, 1982, 1988a; Langeheine \& van de Pol, 1993; Luijkx, 1988; van de Pol \& Langeheine, 1990), as well as the explicit integration of group differences and DIF phenomena that are meaningful for substantive research (e.g., Clogg \& Goodman, 1984, 1985; Kelderman \& Macready, 1990; Mislevy \& Verhelst, 1990; Rost, 1990, 1991; Rost \& von Davier, 1993; Westers \& Kelderman, 1992, 1993). Particular extensions of Rasch models for measuring change have been proposed by Fischer (1983), Fischer \& Formann (1982), Fischer \& Parzer (1991) and Fischer \& Ponocny (1994). Consequently, LSA models can be applied in a wide range of contingency table analyses beyond test construction or test evaluation.

Here, different LSA approaches are discussed for modeling and testing specific hypotheses on latent developmental processes in longitudinal research. For the sake of simplicity, the presentation is confined to one indicator per measurement occasion. However, the generalization to a set of indicators is straightforward. In the case considered here, the manifest variables $X_{1}, \ldots, X_{M}$ that span an $M$-way contingency table refer to repeated observations of a discrete random variable $X$ at $M$ measurement occasions. If a unidimensional $\theta$ is presumed, one static latent variable affects the response behavior at all time points. Nevertheless, latent change may occur that is located in the potential variability of the structural item parameters over time (for models with explicitly dynamic time-related latent variables see Andersen, 1985, 1988, 1991; Collins \& Wugalter, 1992; Embretson, 1991; Langeheine, 1988a, 1993; Langeheine \& van de Pol, 1993; Meiser, in press; van de Pol \& Langeheine, 1990).

Particular longitudinal LSA models result from specifications of the latent variable or vector and of the functional relation between the latent variable(s) and the category probabilities of the indicators. Specification of a unidimensional continuous $\theta$ yields a unidimensional latent trait model. If $\theta$ is discrete, a latent class model results. The concepts of change implied by Rasch latent trait models and by latent class models are rather different: The application of unidimensional Rasch models to longitudinal data assumes homogeneity of change over persons. Using latent class models, different patterns of development can be studied for different classes; thus, heterogeneity of change is assumed. Latent class models, however, do not permit a differentiation between persons of the same latent class. The mixed Rasch model (Kelderman \& Macready, 1990; Rost, 1990, 1991; Rost \& von Davier, 1993) combines the Rasch model and the latent class approach by dividing the population of persons into classes that conform to Rasch models with classspecific parameters. Thus, qualitatively different patterns of change can be modeled and the homogeneity assumption is retained within each class, but is dropped for persons of different classes. The mixed Rasch model also facilitates a quantitative differentiation between persons of the same qualitative developmental pattern by the inclusion of person parameters within the classes.

\section{Rasch Models for the Investigation of Homogeneity of Change}

The general unidimensional Rasch model for polytomous indicators specifies the conditional probability that response $x_{i}$ is observed for person $v$ in variable $X_{i}$ by the function 
$p\left(X_{i}=x_{i} \mid \theta_{v}\right)=\frac{\exp \left(x_{i} \theta_{v}+\sum_{j=1}^{x_{i}} \alpha_{i j}\right)}{\sum_{s=0}^{L-1} \exp \left(s \theta_{v}+\sum_{p=1}^{s} \alpha_{i p}\right)}$, with $\sum_{j=1}^{0} \alpha_{i j}=0$,

where

$L$ is the number of response categories, $L \geq 2$;

$x_{i}$ is one of the $L$ response categories of indicator $X_{i}, x_{i} \in\{0, \ldots, L-1\}$;

$\alpha_{i j}$ is the item-specific easiness parameter of threshold $j, j \in\{1, \ldots, L-1\}$; and

$\theta_{v}$ is the latent parameter of person $v$ (i.e., the realization of $\theta$ ) (Andrich, 1978; Masters, 1982, 1988; Rost, 1988).

Because all LSA models assume conditional stochastic independence, the probability of an observed response vector $\mathrm{y}=\left(X_{1}=x_{1}, \ldots, X_{M}=x_{M}\right)$ given $\theta_{v}$ results from the product of Equation 1 over $i$ :

$p\left(\mathbf{Y}=\mathbf{y} \mid \theta_{v}\right)=\frac{\exp \left(t \theta_{v}+\sum_{i=1}^{M} \sum_{j=1}^{x_{i}} \alpha_{i j}\right)}{\prod_{i=1}^{M} \sum_{s=0}^{L-1} \exp \left(s \theta_{v}+\sum_{p=1}^{s} \alpha_{i p}\right)}$,

where $t$ is the total score of response vector $\mathbf{y}$ (i.e., the sum of the observed response categories $x_{i}$ ). The general unidimensional Rasch model for polytomous indicators (Equation 2) is called the partial credit model (PCM).

From Equation 2, the conditional PCM can be derived by conditioning on the total score variable, which is the sufficient statistic for $\theta_{v}$ :

$$
p(\mathbf{Y}=\mathbf{y} \mid t)=\frac{\exp \left(\sum_{i=1}^{M} \sum_{j=1}^{x_{i}} \alpha_{i j}\right)}{\sum_{\mathbf{y}} \exp \left(\sum_{i=1}^{M} \sum_{p=1}^{x_{i}} \alpha_{i p}\right)},
$$

where the first summation in the denominator is over $\mathrm{y}$ with $\Sigma_{i} x_{i}=t$ (Andersen, 1990; Kelderman, 1984; Masters, 1982; Rost, 1988). This conditional representation of the Rasch model is often preferred because it does not contain $\theta_{v}$. In order to achieve identifiability, one additional constraint must be imposed in Equations 2 and 3 . The usual marginal condition of centered scales is proposed:

$\sum_{i=1}^{M} \sum_{j=1}^{L-1} \alpha_{i j}=0$.

For $M$ repeated observations of a polytomous indicator, differences of threshold parameters between measurement occasions, $\alpha_{i j}-\alpha_{(i+1) j} \neq 0$, indicate that the conditional response probabilities given in Equation 1 have changed. Because this change is formally located in the parameters, it is independent from the respondents and their locations on $\theta$ (Fischer, 1987; Langeheine, 1993; Langeheine \& van de Pol, 1990; Rost, 1989; Spada \& McGaw, 1985, Model I). Therefore, in the unidimensional Rasch model, the process of change is subjected to the assumption of person homogeneity. The model in Equation 2 includes interindividual differ- 
ences in $\theta$, which are stable over time, and intraindividual change, which is identical for all persons.

By the decomposition of the threshold parameters

$$
\alpha_{i j}=\alpha_{j}+\sum_{h=2}^{i} \lambda_{h j},
$$

with $\alpha_{j}=\alpha_{1 j}, \lambda_{h j}=\alpha_{h j}-\alpha_{(h-1) j}$ for $h \geq 2$, and

$$
\sum_{h=2}^{1} \lambda_{h j}=0
$$

into their initial values $\alpha_{j}$ and change parameters $\lambda_{h j}$, which are defined as differences between easiness parameters on consecutive measurement occasions, specific hypotheses about the latent process of development can be specified. For this purpose, additional restrictions can be imposed on the change parameters, such as fixing $\lambda_{h j}$ to 0 for modeling absolute stability or imposing equality constraints on the change parameters over occasions for modeling stationarity of change (i.e., the same amount of change occurs between any consecutive measurement occasions within a particular period of time). Another model follows from constraining the change parameters to be threshold-independent,

$$
\lambda_{h j}=\lambda_{h} \forall j \in\{1, \ldots, L-1\} \text {. }
$$

Equation 7 implies that the interthreshold distances remain unchanged over time

$$
\alpha_{i j}-\alpha_{i j^{\prime}}=\alpha_{i^{\prime} j}-\alpha_{i^{\prime} j^{\prime}} \forall i, i^{\prime} \in\{1, \ldots, M\}, j, j^{\prime} \in\{1, \ldots, L-1\} .
$$

The model in Equation 2 with the restriction in Equation 8 is equivalent to the rating scale model (RSM; Andrich, 1978).

When the data consist of one repeatedly observed indicator, the RSM is important for two reasons. First, the RSM requires that the latent response format of the indicator is invariant over time; thus, it explicitly takes into account that the same indicator is used at all occasions. The PCM (Equation 2) does not do this. Second, only in the RSM are changes of the threshold parameters interpreted as a joint movement of the persons' locations on the latent continuum. Therefore, the RSM facilitates analyzing global change of persons' $\theta$ levels, because $\lambda_{h}$ can be thought of as the global difference in the latent trait between adjacent measurement occasions $h$ and $h-1$. Therefore, the change parameters in the RSM correspond to the "modifiabilities" in Embretson's (1991) extension of the dichotomous Rasch model. The crucial difference is that, in contrast to Embretson's approach, the change parameters are invariant over persons (see also Langeheine, 1993); that is, homogeneity of change is assumed.

In the longitudinal PCM model (Equation 2) without restriction (Equation 8), the $\lambda_{h j}$ parameters cannot be interpreted as directly indicating the amount of change in the $\theta$ s. In many applications, however, this type of change is what is of interest. However, in some cases threshold-specific change can also be meaningful for scientific theory (e.g., if it is assumed that persons acquire new solution strategies from one occasion to the next that alter the relations of subtask easiness in an achievement item). This type of change, however, might raise doubts about the suitability of a structural model based on a unidimensional latent space.

A very general framework of linear decompositions of parameters in polytomous Rasch models was presented by Fischer \& Parzer (1991) and Fischer \& Ponocny (1994). They demonstrated the particular advantages of the approach for measuring change.

\section{Latent Class Analysis for Modeling Heterogeneity of Development}

In latent class analysis (LCA), the latent variable $\theta$ is discrete, $\theta_{v} \in\{1, \ldots, C\}$, dividing the population 
into $C$ mutually exclusive and jointly exhaustive classes. All persons belonging to a certain latent class $g$, $g \in\{1, \ldots, C\}$, have the same probability of responding in category $x_{i}$ of indicator $X_{i}$ :

$\pi_{i x_{i} \mid g}=p\left(X_{i}=x_{i} \mid \theta_{v}=g\right)$,

with

$\sum_{x_{i}=0}^{L-1} \pi_{i_{i} \mid l_{8}}=1$.

The probability of observing response vector $\mathbf{y}=\left(X_{1}=x_{1}, \ldots, X_{M}=x_{M}\right)$ in class $g$ results from Equations 9-10 and conditional independence:

$p(\mathbf{Y}=\mathbf{y} \mid g)=\prod_{i=1}^{M} \pi_{i x_{i} \mid g}$.

The marginal probability of $\mathbf{y}$ is then

$p(\mathbf{Y}=\mathbf{y})=\sum_{g=1}^{c} \pi_{g} \prod_{i=1}^{M} \pi_{i x_{i} \mid g}$,

where $\pi_{g}$ is the proportion of latent class $g$ (Andersen, 1982, 1988, 1990; Dayton \& Macready, 1980; Erdfelder, 1990; Langeheine, 1988b; Lazarsfeld \& Henry, 1968; van de Pol \& Langeheine, 1990). Person $v$ is said to belong to class $g$ if class $g$ shows the highest probability given the person's response vector $\mathbf{y}$; that is, if $p(g \mid \mathbf{y})>p\left(g^{\prime} \mid \mathbf{y}\right)$ for all $g^{\prime} \neq g . p(g \mid \mathbf{y})$ can be calculated using Equations 11 and 12 :

$p(g \mid \mathbf{Y}=\mathbf{y})=\frac{\pi_{g} p(\mathbf{Y}=\mathbf{y} \mid g)}{p(\mathbf{Y}=\mathbf{y})}=\frac{\pi_{g} \prod_{i=1}^{M} \pi_{i x, \mid g}}{\sum_{g=1}^{c} \pi_{g} \prod_{i=1}^{M} \pi_{i, \mid g}}$.

The modal recruitment probability (Equation 13) indicating membership in a certain class can be rather low. For example, in a three-class LCA model $p(1 \mid y)=p(2 \mid y)=.25$ and, consequently, $p(3 \mid y)=.50$ may occur for some $y$. Persons with response vector $y$, therefore, are classified with a very large error rate into the third class. For this reason, classification of persons made using LCA solutions should be regarded with caution. Hagenaars \& Luijkx (1990) presented equations for the evaluation of the strength of association between the latent class variable and the observed response vectors. Often, however, LCA is not used for classification purposes, but for modeling and testing the structure in an empirical dataset.

In order to specify hypotheses about the underlying structure in a given multiway contingency table, the parameters in Equation 12 can be subjected to fixations or equality constraints. In the context of developmental research, probabilistic models of cumulative growth can be specified by appropriate parameter restrictions (e.g., see Henning \& Rudinger, 1985; Schröder, Edelstein, \& Hoppe-Graff, 1991). In addition, the latent classes may be regarded as representing classes with different developmental trajectories. Accordingly, in LCA intraindividual change is assumed to be heterogeneous over latent classes, and no interindividual differentiation is available for persons of the same latent class. As a consequence of the lack of class-internal quantitative differences, qualitative as well as quantitative aspects are mirrored by the division of the population into classes. Thus, qualitative patterns of change and quantitative levels of the persons may be confounded in LCA solutions, as illustrated below. 


\section{Mixture Distribution Rasch Models Representing Distinct Patterns of Homogeneous Development}

The mixture distribution Rasch model may be regarded as an integration of the unidimensional Rasch model (Equation 2) and the LCA rationale (Equation 12). As in LCA, the population is divided into $C$ mutually exclusive classes, but in the mixed Rasch model each class conforms to a Rasch model with class-specific parameters $\theta_{v \mid g}$ and $\alpha_{i j \mid g}$ (Kelderman \& Macready, 1990; Rost, 1990, 1991; Rost \& von Davier, 1993). Consequently, the mixed Rasch model can be derived by rewriting Equation 2 conditional on value $g$ of the discrete latent mixture variable:

$$
p\left(\mathbf{Y}=\mathbf{y} \mid \theta_{v \mid g}, g\right)=\frac{\exp \left(t \theta_{v \mid g}+\sum_{i=1}^{M} \sum_{j=1}^{x_{i}} \alpha_{i j \mid g}\right)}{\prod_{i=1}^{M} \sum_{s=0}^{L-1} \exp \left(s \theta_{v \mid g}+\sum_{p=1}^{s} \alpha_{i p \mid g}\right)},
$$

with

$\sum_{j=1}^{0} \alpha_{i j \mid g}=0 \forall i, g$.

The marginal probability of response vector $\mathbf{y}$ is given by

$$
p(\mathbf{Y}=\mathbf{y})=\sum_{g=1}^{c} \pi_{g} p(\mathbf{Y}=\mathbf{y} \mid g) \text {, }
$$

where $\pi_{g}$ is the proportion parameter of class $g$ as in LCA, and $p(\mathbf{Y}=\mathbf{y} \mid g)$ can be rewritten using the conditional Rasch model in Equation 3 for each class $g$ and the class-specific proportion of total score $t$, $\pi_{t \mid g}$ :

$$
p(\mathbf{Y}=\mathbf{y} \mid g)=p(\mathbf{Y}=\mathbf{y} \mid t, g) \pi_{t \mid g}=\frac{\exp \left(\sum_{i=1}^{M} \sum_{j=1}^{x_{i}} \alpha_{i \mid g}\right)}{\sum_{\mathbf{y}} \exp \left(\sum_{i=1}^{M} \sum_{p=1}^{x_{1}} \alpha_{i p \mid g}\right)} \pi_{t \mid g},
$$

where the first summation in the denominator is over $\mathrm{y}$ with $\sum_{i} x_{i}=t$. The $\pi_{t \mid g}$ are treated as model parameters that are estimated from the data (Rost, 1990, 1991). Thus, no distributional assumptions are necessary in order to obtain the probability of response vector $y$ in Equation 16.

Extreme response patterns with $t=0$ and $t=M(L-1)$ do not contain any information about class membership and their proportions cannot be estimated within a class. Consequently, in the mixed Rasch model there are $C[M(L-1)-2]$ independent $\pi_{t \mid 8}$ parameters and two additional parameters for the proportions of the minimum and the maximum total score patterns. Furthermore, $C-1$ nonredundant class proportions $\pi_{g}$ and $C[M(L-1)-1]$ unconstrained class-specific threshold parameters $\alpha_{i j \mid g}$ must be estimated, unless the number of threshold parameters is reduced by additional restrictions.

Because the extreme response patterns must be excluded from the partition of the population into classes, in the mixed Rasch model the classes are not exhaustive. It follows that the class proportions $\pi_{g}$ do not sum to 1.0 as they do in LCA. Rather, they sum to the proportion of persons with nonextreme observed response patterns.

Parameter estimation using the EM algorithm is discussed by Rost $(1990,1991)$ and implemented in the program MIRA (Rost \& von Davier, 1992). As in LCA, a person is said to be a member of the latent class that 
has the highest recruitment probability given his or her manifest response pattern. The recruitment probabilities are obtained by inserting Equations 16 and 17 into the first ratio of Equation 13. Analogously, the problem of potentially high rates of misclassifications for some response vectors may also arise.

The mixed Rasch model can be regarded as a two-dimensional LSA model with one continuous and one discrete latent variable, because each person is characterized by the combination of membership in latent class $g$ and the individual intraclass parameter $\theta_{v \mid g}$. The unidimensional conditional Rasch model as well as the LCA approach are special cases of this two-dimensional model resulting from Equation 14 by the restrictions $C=1$ and, respectively, $\theta_{v \mid g}=\theta_{\mid g}$ for all persons in class $g$. Therefore, the mixed Rasch model overcomes the major limitations of both hierarchically subordinated model families, the LCA and the Rasch models.

Applied to the longitudinal data situation considered here, the mixed Rasch model allows the study of qualitatively different patterns of change that are represented by the latent classes in combination with quantitative differences of the latent trait within each qualitative pattern of development. In the unidimensional models discussed above, the aspects of quantitative differences in level and qualitative differences in change are either confounded-as in LCA-or one aspect is neglected entirely-as is the possibility of different developmental trajectories in the unidimensional Rasch model. In the mixed Rasch model, the range of the homogeneity assumption concerning the process of intraindividual development is limited to the classes because the time-dependent variability of the threshold parameters is not restricted to be equal over classes. Thus, with the mixed Rasch model it is possible to disentangle quantitative interindividual differences in the latent characteristic under study and qualitative interindividual differences in intraindividual change.

In Equation 14, additional restrictions can be imposed on the conditional threshold parameters in order to specify hypotheses on the response format of the indicator and on the process of change within the classes. The constraints discussed in the context of Equations 5, 7, and 8 are suitable candidates. In the application described below, mixed Rasch models for two latent classes are discussed using the restriction in Equation 8 for each class. These are two-class mixed RSMs. In this case, the interpretation of the longitudinal RSM in terms of global change of the persons' locations applies to each of the classes.

\section{Example: Personality Development During the Transition from Childhood to Early Adolescence}

\section{Method}

The data were part of a reanalysis of the "German Postwar Generation Study" (Thomae, 1965). The study was conducted from 1952 to 1961 and investigated the longitudinal development of children in postwar Western Germany with respect to cognitive variables, personality, school performance, somatic indicators, and social living conditions of the children and their families. Data were assessed from approximately $N=4,000$ children at six research centers. The sample was divided into two cohorts. The younger children were born in 1945/1946 and were assessed on 10 occasions; the older children were born in 1938/ 1939 and were examined on five occasions (1952 to 1956).

Not all data were available for the reanalysis; thus, the analysis was based on a stratified subsample of $N=600$ younger children with uniform proportions in the gender and research center categories.

First, the existence of different latent developmental trajectories was demonstrated for two personality indicators-Activity and Adjustment-using separate mixed Rasch analyses. In the original study, Activity and Adjustment were two of eight personality dimensions that were rated at each measurement occasion from observing the children's overt behavior during the test situations and an interview (Thomae, 1965). For the present analysis, the ratings were transformed into three categories according to the recoding scheme suggested by Uhr (1966; see also Thomae, 1965) where 0 indicated a low degree of Activity or 
Adjustment, 1 indicated a medium degree, and 2 indicated a high degree of Activity or Adjustment. Activity and Adjustment assessed in 1955, 1956, and 1957 were selected as manifest indicators.

Finally, associations of the longitudinal patterns with the external variables school performance, gender, and socioeconomic status were examined in order to evaluate potential predictors and concomitant variables of the latent courses of change. Because of the uncertainty of class assignment to persons or observed response vectors, the cross-classification of class membership in both attributes and discrete exogenous variables were calculated using the explicit recruitment probabilities.

MIRA (Rost \& von Davier, 1992) was used for the computations. A 5\% statistical error rate was used as the boundary for model rejection.

\section{Results}

Developmental trajectories of Activity and Adjustment. The three-way contingency table created by the indicators of Activity and the empirical frequencies $(n)$ of the 27 observable longitudinal patterns are shown in Table 1. Due to missing values, the sample size was reduced to $N=545$. The unidimensional PCM (Equation 2) was rejected using the likelihood ratio test statistic: $G^{2}=30.92$, with 15 degrees of freedom (df), $p<.05$. The mixture distribution PCM (Equation 14) with two latent classes yielded a value of $G^{2}=$ $1.65,5 d f, p>.05$; thus, it fit the data very closely. A two-class mixed RSM (Equation 14 with the restriction of Equation 8) was also found to be compatible with the data: the likelihood ratio test statistic yielded a value of $G^{2}=6.24,9 \mathrm{df}, p>.05$, which indicated that the goodness of fit of the two-class mixed PCM and the two-class mixed RSM did not differ significantly. For reasons of parsimony and interpretability with respect to homogeneous unidimensional change, the restricted mixed RSM with the constraints in Equation 8 per class was preferred.

The expected category probabilities in the longitudinal two-class mixed RSM are shown in Figure 1. The trajectory of Class 1 (Figure la) with an estimated class proportion of approximately $\hat{\pi}_{1}=.29$ indicates that there was almost no change at all, because the expected category probabilities were almost invariant over time. This means that a child's probability of being in a certain category was nearly the same for all measurement occasions in this class. Note, however, that the actual probability depended on the child's latent level and was not completely determined by class membership. Contrary to Class 1, Class 2 (Figure 1b) represents a rather irregular course of development, because at the second point of time children were far more likely to be rated in the highest category than at the other occasions. The proportion of this class was estimated as approximately $\hat{\pi}_{2}=.61$. Thus, qualitatively distinct patterns of development were separated by this longitudinal mixed Rasch model. The class proportions summed to approximately .90 , because the rest of the children had total scores across occasions of 0 or 6; that is, they were persistently rated to be low or high in Activity.

Because the RSM was selected for both latent classes, the result can be interpreted in terms of class-specific global change of person parameters. The estimated threshold parameters are given in Table 2. From these estimates, values of $\hat{\lambda}_{2}=.015$ and $\hat{\lambda}_{3}=.06$ were obtained for the change parameters (see Equations 5 and 7) in Class 1 ; in Class $2, \hat{\lambda}_{2}=3.71$ and $\hat{\lambda}_{3}=-3.63$. From these values and from Figure 1 it was concluded that the $\boldsymbol{\theta}_{v \mid 1}$ parameters were almost completely stable, whereas $\theta_{v \mid 2}$ increased remarkably from the first to the second assessment occasion and decreased again from the second to the third. At the same time, invariance of the underlying rating structure per class is part of the model, because the interthreshold distances remained invariant over time for each class (see Equation 8).

For comparison, the data were also analyzed with unrestricted LCA using PANMARK (van de Pol, Langeheine, \& de Jong, 1991). Only the three-class solution was accepted-the likelihood ratio statistic was $G^{2}=8.88,6 d f, p>.05$. The expected response probabilities are shown in Figure 2 . In the three-class solution, both Class 2 (Figure $2 \mathrm{~b}$ ) and Class 3 (Figure 2c) indicated an increase in the highest category at 
Table 1

Longitudinal Patterns of Activity and Observed Frequencies $(N=545)$

\begin{tabular}{|c|c|c|c|}
\hline \multicolumn{3}{|c|}{ Activity } & \multirow[b]{2}{*}{$n$} \\
\hline$\overline{1955}$ & 1956 & 1957 & \\
\hline 0 & 0 & 0 & 15 \\
\hline 0 & 0 & 1 & 4 \\
\hline 0 & 0 & 2 & 2 \\
\hline 0 & 1 & 0 & 9 \\
\hline 0 & 1 & 1 & 33 \\
\hline 0 & 1 & 2 & 1 \\
\hline 0 & 2 & 0 & 1 \\
\hline 0 & 2 & 1 & 6 \\
\hline 0 & 2 & 2 & 5 \\
\hline 1 & 0 & 0 & 6 \\
\hline 1 & 0 & 1 & 15 \\
\hline 1 & 0 & 2 & 4 \\
\hline 1 & 1 & 0 & 31 \\
\hline 1 & 1 & 1 & 178 \\
\hline 1 & 1 & 2 & 18 \\
\hline 1 & 2 & 0 & 6 \\
\hline 1 & 2 & 1 & 82 \\
\hline 1 & 2 & 2 & 29 \\
\hline 2 & 0 & 0 & 1 \\
\hline 2 & 0 & 1 & 2 \\
\hline 2 & 0 & 2 & 2 \\
\hline 2 & 1 & 0 & 3 \\
\hline 2 & 1 & 1 & 18 \\
\hline 2 & 1 & 2 & 3 \\
\hline 2 & 2 & 0 & 2 \\
\hline 2 & 2 & 1 & 26 \\
\hline 2 & 2 & 2 & 43 \\
\hline
\end{tabular}

the second occasion. These classes represent qualitatively similar trajectories of development and differ primarily in level. Class 1 (Figure 2a) is distinguished from the other classes by qualitative differences in the shape of the developmental process. Nevertheless, Figure 2 illustrates that the classes reflected quantitative differences rather than qualitative patterns of development, because in Class 1 the low category had the highest probability at all occasions, in Class 2 this occurred for the medium category, and in Class 3 for the high category. Class 1 had an estimated proportion of $\hat{\pi}_{1}=.06$, which is very small. The major portion of the population belonged either to Class $2\left(\hat{\pi}_{2}=.72\right)$ or to Class $3\left(\hat{\pi}_{3}=.22\right)$.

In the LCA solution, the classes represent both quantitative and qualitative aspects; however, these are disentangled in the mixed Rasch model. This finding was emphasized when comparing the class-specific expected total scores of response vectors in the two models. In the two-class mixed RSM, the expected scores were very similar-3.0 and 3.37-whereas in the three-class LCA solution the expected scores were $.85,3.09$, and 5.05. These vary over a much broader range. Thus, the advantage of the mixed Rasch model of including quantitative differences of persons within the latent classes becomes obvious: The latent classes were distinguished by mainly qualitative criteria and hardly differed quantitatively. This did not hold true for the LCA solution.

Similar to the analysis of Activity, a two-class mixed RSM was also the appropriate model for the Adjustment ratings $\left(G^{2}=10.29,9 d f, p>.05\right)$. The resulting classes can also be interpreted as representing classes with a rather stable (Class 1 ) and a rather irregular shape of development with an excess in the high 
Figure 1

Class-Specific Expected Category Probabilities in the Two-Class Mixed RSM for Low, Medium, and High Levels of Activity

a. Class 1

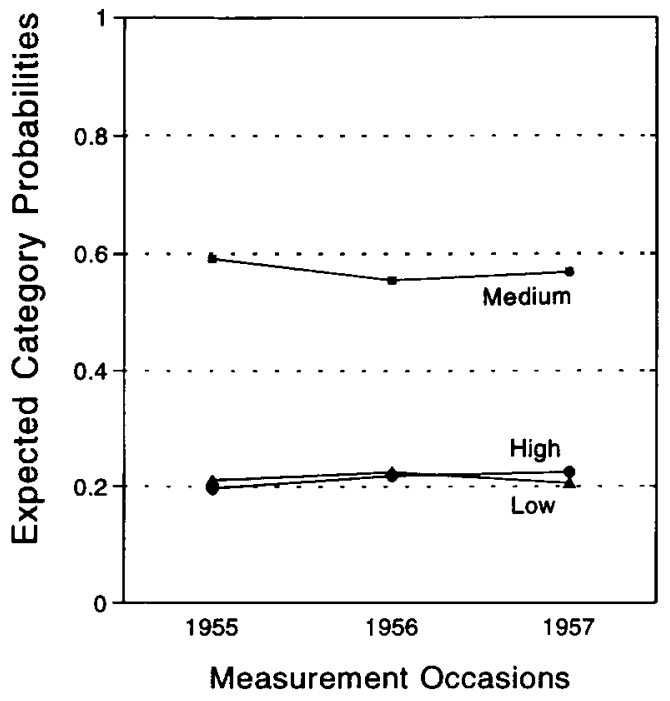

b. Class 2

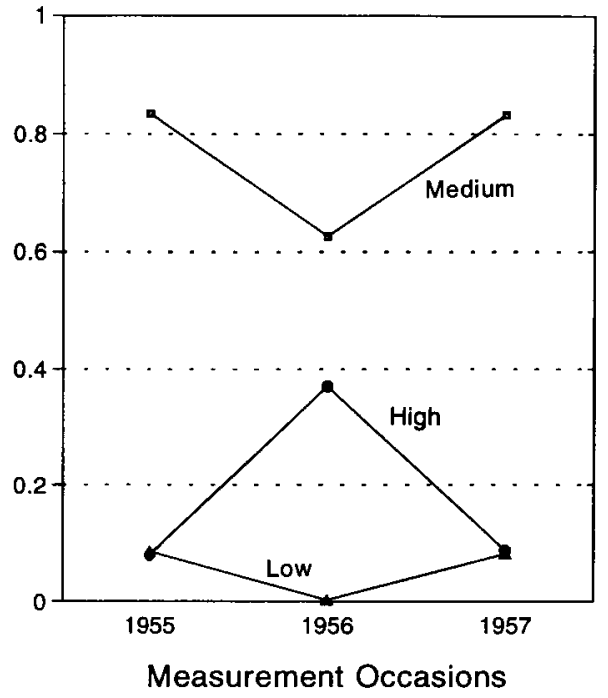

category at the second occasion (Class 2). The unidimensional PCM (Equation 2) was rejected for the Adjustment data, because of a likelihood ratio test statistic of $G^{2}=25.23,15 d f, p<.05$.

Potential predictors of change and concomitant variables. Associations of the longitudinal patterns with external variables were examined in order to evaluate potential predictors and concomitant variables of the latent course of change. Associations were investigated between the latent classes of personality development and discrete external variables, such as gender, school performance, and social indexes. Because of the uncertainty of class assignment of persons or observed response vectors, the cross-classifications of class membership and discrete exogenous variables were calculated using the explicit recruitment probabilities (see Appendix). The shape of development in Activity was not significantly associated with gender (Pearson's $\chi^{2}=2.26,1 d f, p>.05$ ), although there was a tendency for boys to be slightly overrepresented in the class of children showing an excess of Activity at the second occasion. In contrast, class membership in Adjustment was highly related to gender (Pearson's $\chi^{2}=111.57,1 d f, p<.05$ ). Girls were far more likely to show a peak in Adjustment in the middle of the time interval (i.e., to belong to Class 2)

Table 2

Estimated Threshold Parameters in the Two-Class Mixed RSM for the Indicators of Activity

\begin{tabular}{lrrr}
\hline \hline Class and & \multicolumn{3}{c}{ Measurement Occasion } \\
\cline { 2 - 4 } Threshold & 1955 & 1956 & 1957 \\
\hline Class 1 & & & \\
$\quad$ Threshold 1 & .95 & .96 & 1.02 \\
Threshold 2 & -1.01 & -.99 & -.93 \\
Class 2 & & & \\
$\quad$ Threshold 1 & 2.50 & 6.21 & 2.58 \\
Threshold 2 & -5.02 & -1.31 & -4.94 \\
\hline
\end{tabular}




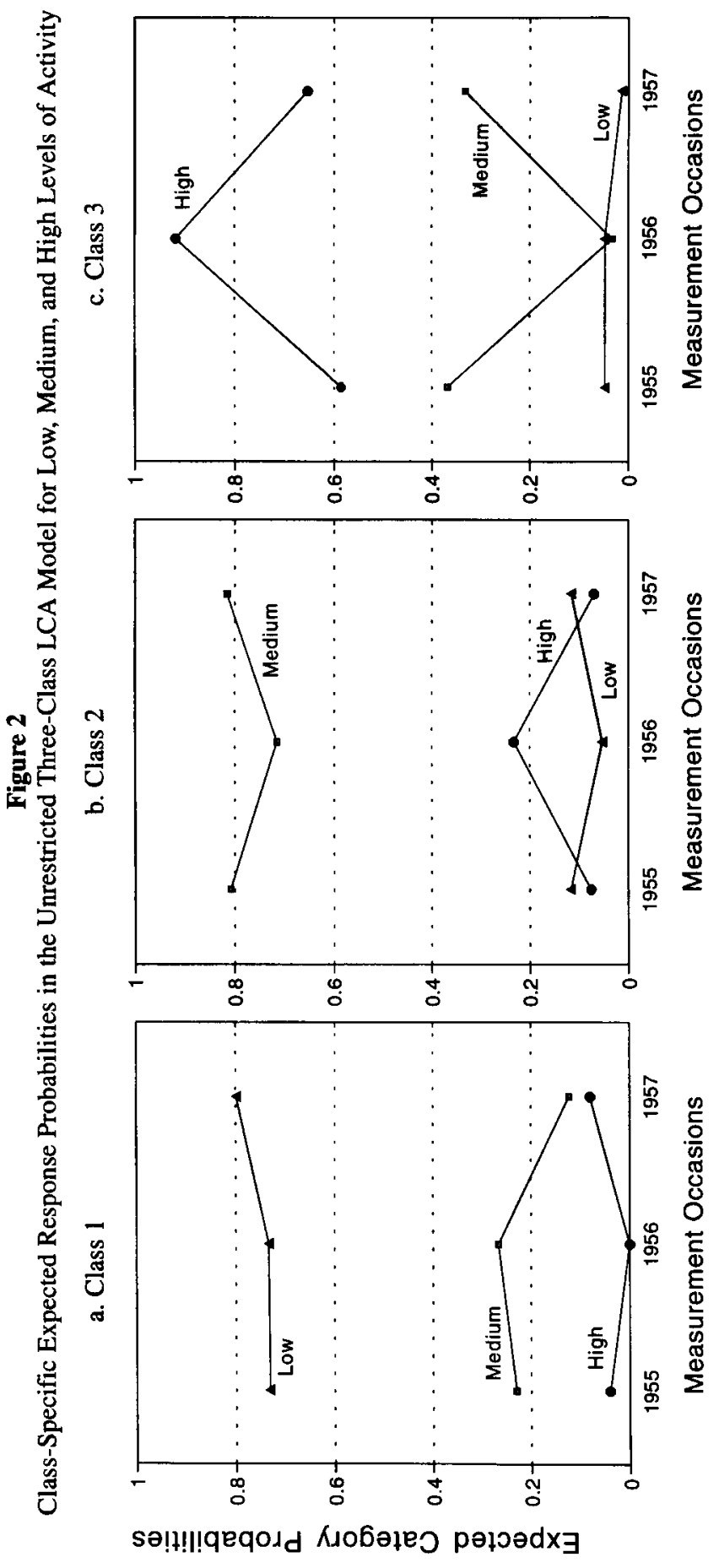

Downloaded from the Digital Conservancy at the University of Minnesota, http://purl.umn.edu/93227. 
than boys. These relations between the shape of personality development and gender correspond with assumptions about gender-specific behavior and coping styles in a period of individual transitions. Thus, these results may be regarded as a tentative validation of the substantive meaning of the mixed RSMs. Indicators of school performance and the families' income (dichotomized by median) were not associated with class membership in either of the personality dimensions.

\section{Discussion}

The mixed Rasch model is a feasible tool in longitudinal research for unmixing qualitatively different patterns of change that are "hidden" in a population. The model overcomes the major limitations of both the Rasch and the latent class approaches by dropping the restrictive assumption of person homogeneity of change for the entire population and by introducing a quantitative differentiation of persons within the same qualitative class.

These theoretical advantages of mixed Rasch models over the usual Rasch and LCA approaches were supported by the results of this study. The two-class mixed Rasch models fit the contingency tables of Activity and Adjustment satisfactorily, whereas the usual Rasch models were rejected. It may be concluded that the existence of qualitatively different developmental trajectories caused the misfit of the unidimensional Rasch models. In terms of probabilistic test theory, the different patterns of change induce differential item functioning or item bias, which violates the basic assumptions of the unidimensional Rasch model but is explicitly part of the mixture distribution approach. In addition, the comparison of the LCA solution and the mixed RSM for the Activity indicator illustrated that in LCA qualitative aspects of development are not clearly disentangled from the quantitative level of Activity; in contrast, the mixed Rasch model does disentangle qualitative from quantitative aspects.

The family of mixture distribution Rasch models appears to be a valuable alternative to unidimensional LSA models for the analysis of longitudinal discrete data. Hypotheses about the process of change can be confirmatorily specified by additional constraints on the class-specific threshold parameters, such as the hypothesis of homogeneous unidimensional quantitative development in each of the classes (the restriction in Equation 8 for each class) or absolute stability for one of the classes (restricting $\lambda_{h j}=0$ in one class). Also, equality constraints of parameters from different classes could and should be considered. In all of these models, the latent classes may be seen as an optimal partition of the total population with respect to the maximum of the loglikelihood of the model, whereas manifest grouping variables may fail to provide an appropriate and powerful splitting in order to detect qualitative heterogeneity (Rost, 1990).

As stated above, the generalization to more than one indicator per occasion is straightforward. In the case of multiple indicators, the Rasch model refers to all of the indicators observed at all of the assessment occasions. By using appropriate parameter restrictions, change parameters of the type in Equations 5 and 6 can be specified that are independent from the thresholds and the items and thus reflect global change of the persons' locations in unidimensional Rasch models (Meiser, in press). If such Rasch models are specified as components in. a finite mixture multinomial model, qualitative differences can be disentangled from the quantitative level of the latent trait as discussed above for the simplified case.

\section{Appendix: Cross-Classification of Class Membership and Discrete Exogenous Variables}

Let $Z$ be a discrete random variable with observed categories $z$. Then, the cross-tabulation of $Z$ and latent class membership in the personality dimension of Activity, for instance, can be written as

$$
\begin{aligned}
\hat{p}\left(g_{\mathrm{ac}}, z\right) & =\hat{p}(Z=z) \hat{p}\left(g_{\mathrm{ac}} \mid z\right)=\hat{p}(Z=z) \sum_{\mathbf{y}_{\mathrm{c}}} \hat{p}\left(g_{\mathrm{ac}}, \mathbf{y}_{\mathrm{ac}} \mid z\right) \\
& =\hat{p}(Z=z) \sum_{\mathbf{y}_{\mathbf{c c}}}\left[\hat{p}\left(g_{\mathrm{ac}} \mid \mathbf{y}_{\mathrm{ac}}, z\right) \hat{p}\left(\mathbf{y}_{\mathrm{ac}} \mid z\right)\right]=\sum_{\mathbf{y}_{\mathrm{ac}}}\left[\hat{p}\left(g_{\mathrm{ac}} \mid \mathbf{y}_{\mathrm{ac}}\right) \hat{p}\left(\mathbf{y}_{\mathrm{ac}}, z\right)\right]
\end{aligned}
$$


The terms in Equation 18 are obtained from either the estimated parameters of the mixed Rasch model [i.e., the recruitment probabilities $\left.\hat{p}\left(g_{\mathrm{ac}} \mid \mathbf{y}_{\mathrm{ac}}\right)\right]$ or from the relative frequencies of response vectors $\left[\mathrm{i} . \mathrm{e} ., \hat{p}\left(\mathbf{y}_{\mathrm{ac}} \mid z\right)\right]$. Here, for each $\mathbf{y}_{\mathrm{ac}}$ both latent classes of Activity enter the calculation of the cross-classification, which is not the case when assigning latent classes to response patterns on the basis of modal recruitment probabilities. Equation 18 is based on a procedure suggested by van de Pol \& Langeheine (1989) in the context of mixed Markov models.

\section{References}

Andersen, E. B. (1982). Latent structure analysis: A survey. Scandinavian Journal of Statistics, 9, 1-12.

Andersen, E. B. (1985). Estimating latent correlations between repeated testings. Psychometrika, 50, 3-16.

Andersen, E. B. (1988). Comparison of latent structure models. In R. Langeheine \& J. Rost (Eds.), Latent trait and latent class models (pp. 207-229). New York: Plenum Press.

Andersen, E. B. (1990). The statistical analysis of categorical data. Berlin: Springer.

Andersen, E. B. (1991). Longitudinal studies for discrete data based on latent structure models. In D. Magnusson, L. R. Bergman, G. Rudinger, \& B. Törestad (Eds.), Problems and methods in longitudinal research: Stability and change (pp. 308-322). Cambridge: Cambridge University Press.

Andrich, D. (1978). A rating formulation for ordered response categories. Psychometrika, 43, 561-573.

Andrich, D. (1991). Review of Rolf Langeheine and Jürgen Rost, Latent trait and latent class models, New York, 1988, Plenum Press. Psychometrika, 56, 155-164.

Clogg, C. C. (1988). Latent class models for measuring. In R. Langeheine \& J. Rost (Eds.), Latent trait and latent class models (pp. 173-205). New York: Plenum Press.

Clogg, C. C., \& Goodman, L. A. (1984). Latent structure analysis of a set of multidimensional contingency tables. Journal of the American Statistical Association, 79, 762-771.

Clogg, C. C., \& Goodman, L. A. (1985). Simultaneous latent structure analysis in several groups. In N. B. Tuma (Ed.), Sociological methodology (pp. 81-110). San Francisco: Jossey-Bass.

Collins, L. M., \& Wugalter, S. E. (1992). Latent class models for stage-sequential dynamic latent variables. Multivariate Behavioral Research, 27, 131-157.

Dayton, M. C., \& Macready, G. B. (1980). A scaling model with response errors and intrinsically unscalable respondents. Psychometrika, 45, 343-356.

Duncan, O. D. (1983). On a dynamic response model of W. F. Kempf. Social Science Research, 12, 393-400.

Duncan, O. D. (1984). Rasch measurement in survey research: Further examples and discussion. In C. F. Turner \& E. Martin (Eds.), Surveying subjective phe- nomena (Vol. II; pp. 367-403). New York: Russell Sage Foundation.

Embretson, S. E. (1991). A multidimensional latent trait model for measuring learning and change. Psychometrika, 56, 495-515.

Erdfelder, E. (1990). Deterministic developmental hypotheses, probabilistic rules of manifestation, and the analysis of finite mixture distributions. In A. von Eye (Ed.), Statistical methods in longitudinal research. Vol. II: Time series and categorical longitudinal data (pp. 471-509). New York: Academic Press.

Fischer, G. H. (1983). Logistic latent trait models with linear constraints. Psychometrika, 48, 3-26.

Fischer, G. H. (1987). Applying the principles of specific objectivity and of generalizability to the measurement of change. Psychometrika, 52, 565-587.

Fischer, G. H., \& Formann, A. K. (1982). Some applications of logistic latent trait models with linear constraints on the parameters. Applied Psychological Measurement, 6, 397-416.

Fischer, G. H., \& Parzer, P. (1991). An extension of the rating scale model with an application to the measurement of change. Psychometrika, 56, 637-651.

Fischer, G. H., \& Ponocny, I. (1994). An extension of the partial credit model with an application to the measurement of change. Psychometrika, 59, 177-192.

Goodman, L. A. (1974a). The analysis of systems of qualitative variables when some of the variables are unobservable. Part I: A modified latent structure approach. American Journal of Sociology, 79, 1179-1259.

Goodman, L. A. (1974b). Exploratory latent-structure analysis using both identifiable and unidentifiable models. Biometrika, 61, 215-231.

Hagenaars, J. A. (1988). LCAG-Loglinear modelling with latent variables: A modified LISREL approach. In W. E. Saris \& I. N. Gallhofer (Eds.), Sociometric research. Vol. 2: Data analysis (pp. 111-130). New York: Macmillan.

Hagenaars, J. A., \& Luijkx, R. (1990). LCAG. A program to estimate latent class models and other loglinear models with latent variables with and without missing data (Working Paper Series 17,2). Tilburg, The Netherlands: Tilburg University, Department of Sociology.

Henning, H. J., \& Rudinger, G. (1985). Analysis of quali- 
tative data in developmental psychology. In $\mathbf{J}$. $\mathbf{R}$. Nesselroade \& A. von Eye (Eds.), Individual development and social change: Explanatory analysis (pp. 295-341). Orlando FL: Academic Press.

Kelderman, H. (1984). Loglinear Rasch model tests. Psychometrika, 49, 223-245.

Kelderman, H. (1992). Computing maximum likelihood estimates of loglinear models from marginal sums with special attention to loglinear item response theory. Psychometrika, 57, 437-450.

Kelderman, H. (1993). Estimating and testing a multidimensional Rasch model for partial credit scoring of children's application of size concepts. In R. Steyer, K. F. Wender, \& K. F. Widaman (Eds.), Psychometric methodology. Proceedings of the 7th European meeting of the Psychometric Society in Trier (pp. 209212). Stuttgart, Germany: Fischer.

Kelderman, H., \& Macready, G. B. (1990). The use of loglinear models for assessing differential item functioning across manifest and latent examinee groups. Journal of Educational Measurement, 27, 307-327.

Kelderman, H., \& Rijkes, C. P. M. (1994). Loglinear multidimensional IRT models for polytomously scored items. Psychometrika, 59, 149-176.

Langeheine, R. (1982). Kausalanalyse qualitatitver Daten mit manifesten und latenten Variablen [Causal analysis of qualitative data with manifest and latent variables]. Zeitschrift für Sozialpsychologie, 13, 163-176.

Langeheine, R. (1988a). Manifest and latent Markov chain models for categorical panel data. Journal of Educational Statistics, 13, 299-312.

Langeheine, R. (1988b). New developments in latent class theory. In R. Langeheine \& J. Rost (Eds.), Latent trait and latent class models (pp. 77-108). New York: Plenum Press.

Langeheine, R. (1993). Diagnosing incremental learning: Some probabilistic models for measuring change and testing hypotheses about growth. Studies in Educational Evaluation, 19, 349-362.

Langeheine, R., \& Rost, J. (Eds.). (1988). Latent trait and latent class models. New York: Plenum Press.

Langeheine, R., \& van de Pol, F. (1990). Veränderungsmessung bei kategorialen Daten [Measuring change in categorical data]. Zeitschrift für Sozialpsychologie, 21, $88-100$.

Langeheine, R., \& van de Pol, F. (1993). Multiple indicator Markov models. In R. Steyer, K. F. Wender, \& K. F. Widaman (Eds.), Psychometric methodology. Proceedings of the 7th European meeting of the Psychometric Society in Trier (pp. 248-252). Stuttgart, Germany: Fischer.

Lazarsfeld, P. F. (1968). Latent structure analysis and test theory. In P. F. Lazarsfeld \& N. W. Henry (Eds.), Readings in mathematical social science (pp. 78-88). Cambridge MA: MIT Press.
Lazarsfeld, P. F., \& Henry, N. W. (1968). Latent structure analysis. Boston: Houghton Mifflin.

Luijkx, R. (1988). Loglinear modelling with latent variables: The case of mobility tables. In W. E. Saris \& I. N. Gallhofer (Eds.), Sociometric research. Vol. 2: Data analysis (pp. 131-159). New York: Macmillan.

Masters, G. N. (1982). A Rasch model for partial credit scoring. Psychometrika, 47, 149-174.

Masters, G. N. (1988). Measurement models for ordered response categories. In R. Langeheine \& J. Rost (Eds.), Latent trait and latent class models (pp. 11-30). New York: Plenum Press.

Meiser, T. (in press). Loglinear Rasch models for the analysis of stability and change. Psychometrika.

Mislevy, R. J., \& Verhelst, N. D. (1990). Modeling item responses when different subjects employ different solution strategies. Psychometrika, 55, 195-215.

Rost, J. (1988). Test theory with qualitative and quantitative latent variables. In $R$. Langeheine \& J. Rost (Eds.), Latent trait and latent class models (pp. 147172). New York: Plenum Press.

Rost, J. (1989). Rasch models and latent class models for measuring change with ordinal variables. In $\mathbf{R}$. Coppi \& S. Bolasco (Eds.), Multiway data analysis (pp. 473-483). Amsterdam, The Netherlands: North Holland.

Rost, J. (1990). Rasch models in latent classes: An integration of two approaches to item analysis. Applied Psychological Measurement, 14, 271-282.

Rost, J. (1991). A logistic mixture distribution model for polychotomous item responses. British Journal of Mathematical and Statistical Psychology, 44, 75-92.

Rost, J., \& von Davier, M. (1992). MIRA-A PC-program for the mixed Rasch model. Kiel, Germany: IPN (Institute for Science Education).

Rost, J., \& von Davier, M. (1993). Measuring different traits in different populations with the same items. In R. Steyer, K. F. Wender, \& K. F. Widaman (Eds.), Psychometric methodology. Proceedings of the 7th European meeting of the Psychometric Society in Trier (pp. 446-450). Stuttgart, Germany: Fischer.

Schröder, E., Edelstein, W., \& Hoppe-Graff, S. (1991). Qualitative analyses of individual differences in intraindividual change: Examples from cognitive development. In D. Magnusson, L. R. Bergman, G. Rudinger, \& B. Törestad (Eds.), Problems and methods in longitudinal research: Stability and change (pp. 166-189). Cambridge: Cambridge University Press.

Spada, H., \& McGaw, B. (1985). The assessment of learning effects with linear logistic test models. In S. Embretson (Ed.), Test design. Developments in psychology and psychometrics (pp. 169-194). Orlando FL: Academic Press.

Thomae, H. (1965). Objective socialization variables and 
personality development. Findings from a longitudinal study. Human Development, 8, 87-116.

Uhr, R. (1966). Verlaufsformen der Entwicklung bei Kindern und Jugendlichen - Ein Beitrag zur differenziellen Jugendpsychologie [Trajectories of development in children and adolescents: A contribution to the differential psychology of adolescence]. Unpublished manuscript, University of Bonn, Department of Psychology, Bonn, Germany.

Van de Pol, F., \& Langeheine, R. (1989). Mixed Markov models, mover-stayer models and the EM algorithm. With an application to labor market data from the Netherlands socioeconomic panel. In R. Coppi \& S. Bolasco (Eds.), Multiway data analysis (pp. 485-495). Amsterdam, The Netherlands: North Holland.

Van de Pol, F., \& Langeheine, R. (1990). Mixed Markov latent class models. In C. C. Clogg (Ed.), Sociological methodology (Vol. 20; pp. 213-247). Oxford: Blackwell.

Van de Pol, F., Langeheine, R., \& de Jong, W. (1991).
PANMARK user manual. Panel analysis using Markov chains-version 2.2. Voorburg, The Netherlands: Netherlands Central Bureau of Statistics.

Westers, P., \& Kelderman, H. (1992). Examining differential item functioning due to item difficulty and alternative attractiveness. Psychometrika, 57, 107-118.

Westers, P., \& Kelderman, H. (1993). Generalizations of the solution-error response-error model (Research Rep. No. 93-1). Twente, The Netherlands: University of Twente, Department of Education.

\section{Acknowledgments}

The authors gratefully acknowledge valuable suggestions from the anonymous reviewers.

\section{Author's Address}

Send requests for reprints or further information to Thorsten Meiser, Psychologisches Institut, Universität Heidelberg, Hauptstr. 47-51, D-69117 Heidelberg, Germany. Internet: thorsten.meiser@urz.uni-heidelberg.de. 